\title{
Goal-Concordant Care in the Era of Advanced Stroke Therapies
}

\author{
Leonard L. Sokol, MD, ${ }^{1,2}$ Joshua M. Hauser, MD, ${ }^{3-5}$ Hillary D. Lum, MD, PhD, ${ }^{6,7}$ Jodi Forlizzi, PhD, ${ }^{8}$ \\ Moran Cerf, PhD, ${ }^{9,10}$ Fan Z. Caprio, MD,11 and Michael J. Young, MD, MPhil ${ }^{12}$
}

\begin{abstract}
Stroke is a leading cause of disability and mortality worldwide. Recent advances in stroke care now enable patients with severe ischemic stroke owing to large vessel occlusion to safely undergo endovascular thrombectomy (EVT) up to 24 hours since their time of last known well, with the goal of improving functional outcomes by recanalization of the occluded vessel and reperfusion of downstream ischemic brain tissue. The objective of this analysis is to highlight clinical and ethical challenges related to ensuring goal-concordant care in this era of unprecedented advances in acute stroke care. Specifically, there is a salient challenge of whether advanced therapies such as EVT may be justifiably considered comfort focused, given their potential to preempt accumulated neurologic disability and suffering at the end of life. Through the lens of a patient case, we discuss key challenges, lessons learned, and suggestions for future care and research endeavors at the intersection of acute stroke care and palliative care principles. Although therapies such as thrombolysis and EVT may be considered aggressive prima facie, their potential to ameliorate additional disability and potential suffering at the end of life prompt close consideration of the proper role of these therapies on a case-by-case basis in the context of comfortfocused care. Modification to the workflow for EVT evaluations may facilitate goal-concordant care and timely resource allocation, especially for cases that involve hospital-to-hospital transfers for advanced stroke care.
\end{abstract}

Keywords: advance care planning, goals of care, hospital transfers, stroke, thrombectomy, withdrawal of interventions

\section{Introduction}

$S^{\mathrm{r}}$ TROKE IS A LEADING SOURCE of disability and mortality in the world, second only to heart disease. ${ }^{1}$ Although intravenous thrombolysis with tissue plasminogen activator (tPA) is the mainstay of acute ischemic stroke therapy, only approximately $5 \%$ of patients are eligible for such treatment. ${ }^{1}$
Time limitations and other narrow inclusion criteria may contribute to low eligibility, as under present guidelines clinicians can only administer tPA if patients present within 4.5 hours since their last known well time. ${ }^{2}$ Unlike tPA, which delivers a systemic blood-thinning medication to alleviate cerebral clot burden, the goal of endovascular thrombectomy (EVT) is the mechanical removal of a clot

\footnotetext{
${ }^{1}$ The Ken and Ruth Davee Department of Neurology, ${ }^{2}$ McGaw Bioethics Scholars Program, Center for Bioethics and Humanities, ${ }^{3}$ Center for Bioethics and Medical Humanities, Institute for Public Health and Medicine, ${ }^{4}$ Section of Palliative Medicine, Department of Medicine, ${ }^{11}$ Division of Stroke and Neurocritical Care, The Ken and Ruth Davee Department of Neurology, Northwestern University Feinberg School of Medicine, Chicago, Illinois, USA.

${ }^{5}$ Palliative Care Service, Jesse Brown VA Medical Center, Chicago, Illinois, USA.

${ }^{6}$ Division of Geriatric Medicine, University of Colorado School of Medicine, Aurora, Colorado, USA.

${ }^{7}$ Eastern Colorado VA Geriatric Research Education and Clinical Center, Rocky Mountain Regional VA Medical Center, Aurora, Colorado, USA.

${ }^{8}$ Human-Computer Interaction Institute, School of Computer Science, Carnegie Mellon University, Pittsburgh, Pennsylvania, USA.

${ }^{9}$ Interdepartmental Neuroscience Program, Northwestern University, Evanston, Illinois, USA.

${ }^{10}$ Kellogg School of Management, Northwestern University, Evanston, Illinois, USA.

Northwestern University Feinberg School of Medicine, Chicago, Illinois, USA.

${ }^{12}$ Department of Neurology, Harvard Medical School, Massachusetts General Hospital, Boston, Massachusetts, USA.

Accepted April 22, 2020.
}

(C) Leonard L. Sokol et al., 2020; Published by Mary Ann Liebert, Inc. This Open Access article is distributed under the terms of the Creative Commons License (http://creativecommons.org/licenses/by/4.0), which permits unrestricted use, distribution, and reproduction in any medium, provided the original work is properly credited. 
to recanalize the occluded vessel and reperfuse downstream ischemic brain tissue. The use of EVT has revolutionized the acute treatment of stroke, as such intervention can occur within 24 hours after the patient's last known well time. ${ }^{3-5}$

Novel clinical and ethical challenges arise in the context of ensuring goal-concordant care as acute stroke care advances, including the challenge of whether advanced therapies such as EVT may be justifiably considered comfort focused given the potential to prevent significant neurological disability and suffering at the end of life. Although therapies such as thrombolysis and EVT may be considered aggressive prima facie, their potential to reduce poststroke suffering at the end of life warrants careful consideration of the potential palliative benefit as part of comfort-focused care. To illustrate these challenges, we discuss the case of a patient with a large vessel occlusion of the right middle cerebral artery (MCA), transferred for an EVT evaluation, for whom the medical team learned only after the transfer that the patient's preferences were for comfort-focused treatments. We consider the value of exploring goals of care before hospital transfers and further define the normative valence of novel acute stroke therapies such as EVT within operationalized treatment categories (i.e., full treatment, selective treatment, and comfortfocused treatment).

\section{Case Description}

A previously healthy 85-year-old left-handed man developed sudden-onset left facial droop, left arm weakness, global aphasia, and rightward gaze deviation. Minutes after the onset of symptoms, emergency services were activated, and transferred the patient to the nearest hospital, where he was diagnosed with an ischemic stroke localizable to the right MCA territory. As the patient was within the 4.5-hour window of his last known well and had no contraindications, he immediately received $\mathrm{tPA}$.

He was transferred to a tertiary care center for consideration of EVT. The patient's examination upon arrival to the tertiary center was unchanged with a National Institutes of Health Stroke Scale (NIHSS) of 20, indicating a severe right MCA syndrome. CT angiography demonstrated a thrombus in the proximal, right MCA; perfusion imaging indicated a sizeable ischemic penumbra (i.e., a vast territory of brain tissue at risk of further irreversible damage if blood flow is not achieved).

The patient was widowed, had one daughter, and no designated health care power of attorney or other advance directives. The medical team reached the daughter, his default surrogate decision maker (surrogate), through phone. This process explored the daughter's understanding of the situation and informed her of the possibility of surgical intervention. The daughter understood that her father had had a stroke and was transported to a different hospital.

The series of diagnostic and therapeutic measures were briefly reviewed, including the use of tPA. She was informed that the stroke had affected a large vessel within the brain that governed functions such as attention, articulation, swallowing, and left-sided sensation and movement. The daughter indicated that at no point had she consented to tPA on her father's behalf (in emergency care settings, a model of pre- sumed consent for emergency therapies, such as tPA, is common), ${ }^{6,7}$ and was unclear why her father was transferred. The medical team underscored that, although tPA was one type of acute stroke therapy, EVT could also potentially remove the clot, possibly decreasing his neurologic deficits and preventing further disability.

His daughter highlighted that several months before his admission he had expressed a preference for a do-notresuscitate (DNR) order when admitted to the hospital for viral gastroenteritis. It was clarified that DNR is not equivalent to "do not treat" or even synonymous with only comfort-focused treatment, ${ }^{8}$ and that one could still have a DNR order and receive medical interventions to prolong or sustain life. Nevertheless, his daughter unequivocally indicated that her father should not receive any medical interventions aimed at prolonging life, in line with her understanding of his prior wishes. She predicated this on his recent behaviors and conversations, including his refusal to take anticoagulation despite a cardiologist's recommendations, declining a pacemaker a year before his stroke, and consistent opposition to medical procedures. In line with this understanding of the patient's preferences and goals, he was treated for symptoms of discomfort and transitioned home with hospice services. The palliative medicine service was consulted, and also ensured that the surrogate participated in informed shared decision making with proper understanding, appreciation, and communication around the reasoning for forgoing life-sustaining treatments. ${ }^{8}$

\section{Discussion}

EVT evaluations are unique, as they often involve a partnership between academic and community clinicians, through virtual interactions like telestroke, and sometimes involve hospital transfers. Because potentially appropriate patients often have very disabling deficits, as few as $17 \%$ of patients may be able to provide consent. ${ }^{9}$ In patients eligible for EVT, there are limited data estimating the proportion of cases where patients or surrogates forgo the intervention and why.

In this case, because the patient exhibited nearly all the clinical attributes requisite for an EVT, the medical team initially responded skeptically to the surrogate's judgment that her father would not want this intervention. This sentiment likely arose from two factors. First, between $34 \%$ and $44 \%$ of patients who undergo EVT experience disability reduction, whereas only $1 \%$ suffer a worsening of disability. ${ }^{10}$ Second, the refusal of EVT appeared contrary to the preceding therapeutic momentum. The patient had first been rushed to the community hospital, received tPA, and then finally transferred to an academic center for EVT. Only at that point was the surrogate asked about goals of care, and compellingly voiced that the patient preferred comfortfocused treatments and would be opposed to any procedure. Team members worked to ensure that the surrogate's articulated choice was consistent with the patient's long-standing values, preferences, and goals.

This case illustrates four salient challenges. First, no formalized advance care planning documentation or portal medical orders, such as a Physician's Orders for Life-Sustaining Treatment (POLST) form, were available, which may have facilitated goal-concordant care from the onset of symptoms. 
The challenges associated with ensuring compassionate, goalconcordant care for patients who are incapacitated are many and amplified in settings where conflicts might exist between clinician perspectives and the substituted judgment of the surrogate. ${ }^{11}$ Second, the case was medically appropriate for EVT, which may have generated erroneous assumptions about the patient's preferences and impeded optimal communication. Third, exploration of the patient's goals of care as part of clinical decision making for advanced stroke treatment and consideration of EVT occurred after transfer. Because the patient's preferences were unknown before the transfer, there was delayed achievement of goal-concordant care and corresponding inappropriate allocation of resources. Fourth, the choice of comfort-focused treatment did not necessarily preclude the use of EVT. ${ }^{12}$ This case highlights the lack of clarity and consensus about whether EVT, as an invasive procedure, can be justifiably conceptualized as promoting comfort.

In discussing the fourth challenge, it is helpful to reference the National Institute on Aging's definition of comfort care. ${ }^{12}$ It defines it as " ...care that helps or soothes a person who is dying... to prevent or relieve suffering as much as possible and to improve quality of life while respecting the dying person's wishes." 13 Scholars have commented on the ambiguity of the phrase, as invasive measures may still help to achieve these aims. ${ }^{12}$ Indeed, EVT is a fitting example. Successful EVT often results in the reduction of aphasia or pain, the return of strength or cognition, and thus may improve quality of life, analogous to the use of palliative radiation. Although the surrogate argued against all interventions for the patient, other patients with a similar clinical presentation and preferences for comfort-focused treatment could foreseeably and justifiably agree to EVT.

Consider, for example, a cancer patient with an estimated 1-year prognosis who has decided to focus on comfort. However, shortly after that decision, the patient suffers a right MCA stroke syndrome. In the appropriate context, the clinical role of EVT could become a question of whether that person would want to live the remaining time paralyzed and aphasic, or with minimal deficits.

Taken together, the four challenges may be surmounted with a modification to the workflow for EVT evaluation. Aligned with other proposals regarding informed consent for EVT, we also suggest that any member from the medical team, including a nurse, social worker, or chaplain, should discuss the potential of advanced stroke therapies with the surrogate as early as the patient arrives with manifestations consistent with a large vessel occlusion. ${ }^{14}$ Miscommunication contributed to $60 \%$ of inappropriate hospital transfers, and data suggest that interaction with the surrogate impacts medical decision making. ${ }^{15}$ Thus, before a transfer to another hospital for EVT, timely communication with the surrogate ought to be a priority.

Time is of the essence in large vessel occlusion strokes, wherein it is estimated that approximately two million neurons are lost for every minute delay in treatment. ${ }^{10}$ Clinicians must, therefore, balance high-quality consent without harming the patient or surrogate. One component that may be helpful is a communication framework for emergency surgical interventions. ${ }^{16}$ Dialogue conveyed from the medical team would include themes such as prognosis, information, options, and goals. ${ }^{16}$ A team member may assess the surrogate's understanding of the situation (information) and then inquire about the surrogate's substituted judgment (goals). The inquiry might be phrased as follows: "What would the individual think/say about having an intervention to remove the clot which might restore function?' Past data demonstrate benefits in using the words "think" or "say" over "want" in this context."

Another component that may enhance the informed consent process is the use of a decision aid. ${ }^{10}$ Decision aids, also commonly used during consent for thrombolytic therapy for acute ischemic stroke, may be delivered electronically or in-person. The optimal visual aid would capture the immediate complications (e.g., dissection and hemorrhagic conversion) and high-level, long-term benefits/risks while avoiding jargon. ${ }^{10}$ Such a tool, which adheres to the guidelines and best practices for consent during emergency scenarios, has already been engineered. ${ }^{10}$ This two-pronged approach-a communication framework and a decision aid-may help to ensure that the patient's values are optimally captured and honored, and further prospective studies to evaluate goal concordance in the acute stroke setting can strengthen paradigms of care. ${ }^{18}$

In the optimal scenario, advance care planning documentation would have been available and easily accessible. Ideally, the medical team would have been able to contact the surrogate, review the patient's stated preferences, and clarify whether EVT was aligned with promoting the patient's goals. Such measures may also obviate the misallocation of resources, which in the forgoing case was only realized in retrospect. Even if advance care planning documentation were available, there still would be appropriate tolerance for a hospital transfer from home to ensure comfort. Indeed, POLST forms permit such malleability as aligned with comfort-focused care. ${ }^{15}$

A reconsideration of the workflow can help safeguard the appropriateness of acute stroke transfers. ${ }^{19}$ Because only limited tertiary centers perform EVTs, further longitudinal studies are needed to explore how to ascertain a patient's values rapidly and ensure goal-concordant care. Decision support tools can use or infer patient preferences based on historical information, classification to similar patients (in the absence of information), or impute data about patients based on extrapolation of existing information. ${ }^{20-22}$ These are likely to yield outcomes that are more aligned with expected patient preferences. ${ }^{23}$

However, these systems cannot take into account the essential human judgments that surround care, primarily from the clinician's perspective. ${ }^{24}$ They raise ethical questions about the tradeoffs between individual, institutional, and societal benefits and the costs of using technology to improve human judgments. That is, whether the scenario is one where the heuristics fall short of the individual's performance, and that is the reason that the individual prefers not to use its recommendation. Alternatively, inherent biases exist against technological solutions-irrespective of the actual performance-that lead stakeholders to refrain from using them.

Future work also ought to explore the number of hospitalto-hospital transfers that involve an escalation of care, those that independent evaluators might judge as at odds with patient preferences, and processes to prevent potentially unwanted transfers. Although therapies such as thrombolysis and EVT may be considered aggressive prima facie, their 
potential to improve quality of life and ameliorate further suffering at the end of life warrants closer consideration of the proper role of these therapies in the context of comfortfocused care on a case-by-case basis.

\section{Authors' Contributions}

L.L.S.: research project: conception, organization, execution; article: writing of the first draft, review, and critique. All other authors: research project: organization, execution; article: review, and critique.

\section{Acknowledgments}

L.L.S. extends his gratitude toward Jonna Peterson for her assistance in identifying pertinent articles.

\section{Funding Information}

L.L.S. is an ad hoc consultant for Tikvah for Parkinson's and receives financial support from Northwestern's Physician-Scientist Training Program for Neurology. H.D.L. is supported in part by the National Institutes of Health (K76AG054782). J.M.H. is supported or has received support from the Coleman Foundation, University of Florida, Instituto Nacional de Câncer, American Academy of Hospice and Palliative Medicine, Arnold P. Gold Foundation, National Institute on Aging, Seasons Hospice Foundation, Woodstock, HCSC Insurance Services Company, National Heart, Lung, and Blood Institute, Canadian Patient Safety Institute, Health Research and Educational Trust, Icahn School of Medicine at Mount Sinai, Centers for Medicare and Medicaid Services, Agency for Healthcare Research and Quality, Children's Hospitals and Clinics of Minnesota, Instituto Nacional de Câncer, Department of Veterans Affairs, National Center for Research Resources, NOVA Research Company, Instituto Nacional de Câncer, Lance Armstrong Foundation, Retirement Research Foundation, Society for the Arts in Healthcare, Medical College of Wisconsin, and the National Institute of Nursing Research. J.F. reports no relevant disclosures. M.C. reports no relevant disclosures. F.Z.C. reports no relevant disclosures. M.J.Y. reports no relevant disclosures.

\section{Author Disclosure Statement}

No competing financial interests exist.

\section{References}

1. Donnan GA, Fisher M, Macleod M, Davis SM: Stroke. Lancet 2008;371:1612-1623.

2. Powers WJ, Rabinstein AA, Ackerson T, et al.: Guidelines for the early management of patients with acute ischemic stroke: 2019 update to the 2018 guidelines for the early management of acute ischemic stroke: A guideline for healthcare professionals from the American Heart Association/American Stroke Association. Stroke 2019;50: e344-e418.
3. Nogueira RG, Jadhav AP, Haussen DC, et al.: Thrombectomy 6 to 24 hours after stroke with a mismatch between deficit and infarct. N Engl J Med 2018;378: $11-21$.

4. Albers GW, Marks MP, Kemp S, et al.: Thrombectomy for stroke at 6 to 16 hours with selection by perfusion imaging. N Engl J Med 2018;378:708-718.

5. Campbell BCV, Mitchell PJ, Kleinig TJ, et al.: Endovascular therapy for ischemic stroke with perfusionimaging selection. N Engl J Med 2015;372:1009-1018.

6. Mendelson SJ, Mark Courtney D, Gordon EJ, et al.: National practice patterns of obtaining informed consent for stroke thrombolysis. Stroke 2018;49:765-767.

7. White-Bateman SR, Schumacher HC, Sacco RL, Appelbaum PS: Consent for intravenous thrombolysis in acute stroke. Arch Neurol 2007;64:785.

8. Holloway RG, Arnold RM, Creutzfeldt CJ, et al.: Palliative and end-of-life care in stroke: A statement for healthcare professionals from the American Heart Association/ American stroke association. Stroke 2014;45:18871916.

9. Qureshi AI, Gilani S, Adil MM, et al.: Pattern of informed consent acquisition in patients undergoing emergent endovascular treatment for acute ischemic stroke. J Vasc Interv Neurol 2014;7:21-25.

10. Tokunboh I, Montero MV, Almeida MFZ, et al.: Visual aids for patient, family, and physician decision making about endovascular thrombectomy for acute ischemic stroke. Stroke 2018;49:90-97.

11. Young M: Compassionate care for the unconscious and incapacitated. Am J Bioeth 2020;20:55-57.

12. Kelemen AM, Groninger $\mathrm{H}$ : Ambiguity in end-of-life care terminology-what do we mean by "comfort care?" JAMA Intern Med $2018 ; 178: 1442-1443$.

13. Providing Care and Comfort at the End of Life: www.nia.nih.gov/health/providing-comfort-end-life (last accessed December 1, 2019).

14. Chen M, Connors J: Blank space. J Neurointerv Surg 2015; 7:391-392.

15. Unroe KT, O'Kelly Phillips E, Effler S, et al.: Comfort measures orders and hospital transfers: Insights from the OPTIMISTIC demonstration project. J Pain Symptom Manage 2019;58:559-566.

16. Cooper Z, Koritsanszky LA, Cauley CE, et al.: Recommendations for best communication practices to facilitate goal-concordant care for seriously ill older patients with emergency surgical conditions. Ann Surg 2016;263:1-6.

17. Schwarze ML, Campbell TC, Cunningham T V., White DB, Arnold RM: You can't get what you want: Innovation for end-of-life communication in the intensive care unit. Am J Respir Crit Care Med 2016;193:14-16.

18. Halpern SD: Goal-concordant care-Searching for the Holy Grail. N Engl J Med 2019;381:1603-1606.

19. Young M, Regenhardt R, Leslie-Mazwi T, Stein M. Disabling stroke in the already disabled: Ethical dimensions and directives. Neurology 2020;94:306-310.

20. Belle A, Thiagarajan R, Soroushmehr SMR, et al.: Big data analytics in healthcare. Biomed Res Int 2015;2015: 370194.

21. Islam MS, Hasan MM, Wang X, et al.: A systematic review on healthcare analytics: Application and theoretical perspective of data mining. Healthcare (Basel, Switzerland) 2018;6:E54. 
22. Levy J, Markell D, Cerf M: Polar similars: Using massive mobile dating data to predict dating preferences. Front Psychol 2019;10:2010.

23. Yang Q, Steinfeld A, Zimmerman J: Unremarkable AI: Fiting intelligent decision support into critical, clinical decision-making processes. In: Conf Hum Factors Comput Syst-Proc, 2019.

24. Longoni C, Bonezzi A, Morewedge CK: Resistance to medical artificial intelligence. J Consum Res 2019;46: 629-650.
Address correspondence to:

Leonard L. Sokol, MD

The Ken and Ruth Davee Department of Neurology

Feinberg School of Medicine

Northwestern University

710 N. Lake Shore Drive

Chicago, IL 60611

USA

E-mail: leonard.sokol@northwestern.edu 